\title{
Development of ultrafine grained Al 7075 by cryogenic temperature large strain extrusion machining - CORRIGENDUM
}

\author{
Xiaolong Yin, Yunyun Pi, Di He, Jiayang Zhang, and Wenjun Deng \\ doi: https://doi.org/10.1557/jmr.2018.313, Published by Materials Research Society with \\ Cambridge University Press, 20 September 2018.
}

In the original publication of Yin et al., ${ }^{1}$ author Di $\mathrm{He}$ was inadvertently omitted from the author list. The article has been updated to reflect the correct authorship. The authors apologize for this error.

\section{REFERENCE}

1. X. Yin, Y. Pi, D. He, J. Zhang, and W. Deng: Development of ultrafine grained Al 7075 by cryogenic temperature large strain extrusion machining. J. Mater. Res. (2018). doi: 10.1557/ jmr.2018.313. 